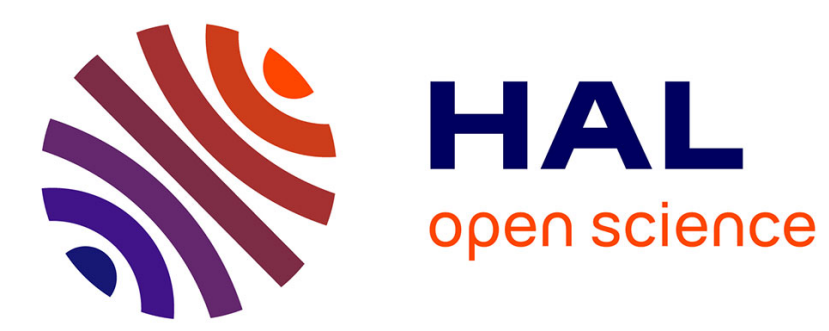

\title{
Litteralior. L'englobement du spirituel dans le littéral selon Pierre de Jean Olivi \\ Sylvain Piron
}

\section{To cite this version:}

Sylvain Piron. Litteralior. L'englobement du spirituel dans le littéral selon Pierre de Jean Olivi . Annali di Scienze Religiose, 2014, 7, pp.179-195. halshs-01313620

\section{HAL Id: halshs-01313620 \\ https://shs.hal.science/halshs-01313620}

Submitted on 10 May 2016

HAL is a multi-disciplinary open access archive for the deposit and dissemination of scientific research documents, whether they are published or not. The documents may come from teaching and research institutions in France or abroad, or from public or private research centers.
L'archive ouverte pluridisciplinaire HAL, est destinée au dépôt et à la diffusion de documents scientifiques de niveau recherche, publiés ou non, émanant des établissements d'enseignement et de recherche français ou étrangers, des laboratoires publics ou privés. 
Sylvain Piron

Litteralior.

L'englobement du spirituel dans le littéral selon Pierre de Jean Olivi

[paru in Annali di scienze religiose, 7, 2014, p. 179-195]

En 1324, Jacques Fournier, qui n'était encore que simple évêque de Pamiers, fut chargé par Jean XXII d'examiner l'orthodoxie de quelques extraits de la Lectura super Apocalipsim de Pierre de Jean Olivi ${ }^{1}$. De tous les experts sollicités par le pape, c'est lui qui en a proposé la réfutation la plus étendue et la plus détaillée. Ce document, premier d'une série d'importantes consultations théologiques remises au pape, a joué un rôle déterminant dans son ascension à la curie, jusqu'à son élection comme successeur de Jean XXII ${ }^{2}$. Pour avoir assisté à la sentence rendue à Pamiers par Bernard Gui contre des béguins du diocèse voisin de Mirepoix, Jacques Fournier connaissait de première main leur doctrine. Il savait que leur inspiration majeure provenait précisément de ce commentaire de l'Apocalypse ${ }^{3}$. L'activité de [180] ce groupe, particulièrement dangereux à ses yeux, explique certainement l'énergie qu'il mit à identifier l'origine de l'hérésie des béguins dans le texte d'Olivi. Son rapport présente donc une grande importance historique. Nous n'en connaissons malheureusement que deux longs extraits, conservés dans un manuscrit de la Bibliothèque municipale d'Avignon ${ }^{4}$. De façon très adroite, Fournier a pris la décision de

\footnotetext{
${ }^{1}$ Pour une vue d'ensemble sur l'histoire de ce procès, je me permets de renvoyer à mes articles: "Bonagrazia de Bergame, auteur des Allegationes sur les articles extraits par Jean XXII de la Lectura super Apocalipsim d'Olivi, in A. Cacciotti, P. Sella (cur.), Revirescunt chartae, codices, documenta, textus. Miscelleana investigationum medioevalium in honorem Caesaris Cenci OFM collecta, Roma, 2002, vol. 2, pp. 10651087 : Censures et condamnation de Pierre de Jean Olivi : enquête dans les marges du Vatican, in Mélanges de l'Ecole française de Rome. Moyen Age, 118/2, 2006, p. 313-373 ; Un avis retrouvé de Jacques Fournier, in Médiévales, 54, 2008, pp. 113-134; La consultation demandée à François de Meyronnes sur la Lectura super Apocalipsim, in Oliviana 3, 2009 [en ligne] http://oliviana.revues.org/330

2 J. Koch, Der Kardinal Jacques Fournier (Benedikt XII) als Gutachter in theologischen Prozessen (1960), in Id., Kleine Schriften, Rome, 1973, t. 2, p. 368-386.

${ }^{3}$ Cf. Le livre des sentences de l'inquisiteur Bernard Gui, 1308-1323, ed. A. PalesGobilliard, Paris, 2002, p. 1308, 1350, 1354, 1396 et 1632.

${ }^{4}$ Pour l'identification du texte, voir Un avis retrouvé de Jacques Fournier, p. 115.
} 
s'attaquer à Olivi en le confrontant à sa principale source, Joachim de Fiore. De ce dernier, l'évêque de Pamiers connaît et utilise principalement la Concordia. Critiquant la façon dont Joachim cherche à fonder la prééminence du sixième âge de l'Eglise au moyen d'une concordance avec différents épisodes bibliques, et notamment le sixième jour de la création, il formule un jugement plus global sur la méthode des deux exégètes :

\begin{abstract}
... Ex mistica teologia voluerunt trahere argumentum, que tamen argumentabilis non est, et volunt adaptare quasi omnes sex sacre scripture ad sextum tempus ecclesie quem finxerunt, cum non possent invenire in scriptura claras probationes ad illud quod ponere de suo capite intendebant ${ }^{5}$
\end{abstract}

Autrement dit, Olivi et Joachim auraient outrepassé les limites de l'allégorie. Au lieu de rapporter au seul Christ les préfigurations typologiques de l'Ancien Testament, ils ont projeté sur le texte biblique le fruit de leur imagination en y cherchant la confirmation d'un hypothétique renouveau de l'Eglise dans un nouvel âge. Soumise à examen, l'exégèse qu'ils produisent des passages sollicités ne se révèle pas fondée de manière non équivoque dans l'Ecriture sainte. Leur herméneutique reposerait sur un cercle vicieux. La clé d'interprétation qu'ils ont forgé leur permet de trouver une vérification presque automatique de leur théologie de l'histoire, en comprenant en un sens mystique les lieux bibliques qui leur conviennent. C'est ainsi qu'ils « veulent appliquer presque tous les six de l'Ecriture sainte au sixième temps de l'Eglise qu'ils ont imaginé ». [181]

La critique de Jacques Fournier est particulièrement intéressante à considérer. En effet, au Moyen Âge central, l'exégèse biblique a très rarement fait l'objet d'une condamnation doctrinale. Seules ont été censurées par Innocent III les propositions dogmatiques de Joachim de Fiore sur la Trinité et non ses travaux exégétiques. Alors que la spéculation théologique est soumise à un contrôle de plus en plus sévère à partir du dernier tiers du treizième siècle, l'interprétation de l'Ecriture sainte semble constituer une sphère de liberté préservée. La principale raison de cet état de fait tient au poids accordé à la tradition interprétative et aux autorités patristiques, qui limite structurellement toute possibilité d'innovation délibérée. De plus, les occasions d'introduire dans la discussion des opinions philosophiques contestables étaient bien moindres. Mais on peut également invoquer un élément strictement épistémologique. L'énoncé dogmatique est par principe univoque; chaque déformation qu'on lui impose produit nécessairement un énoncé fautif qui pourra être qualifié d'hérétique si l'erreur est maintenue avec obstination. En revanche, le

\footnotetext{
${ }^{5}$ Avignon, Bibliothèque municipale, cod. 1087, f. 169v.
} 
travail d'interprétation textuelle autorise une plus grande flexibilité. La pluralité des sens de l'Ecriture impose de tolérer une multiplicité de solutions compatibles entre elles. La démonstration qu'une exégèse est déliberemment fautive et qu'elle mérite la qualification d'hérésie est bien plus complexe à produire. Les difficultés qu'a rencontrées Jean XXII pour condamner la Lectura super Apocalipsim, après huit années de procédure, en fournissent une bonne illustration.

Sollicité en même temps que Jacques Fournier sur ce dossier, en 1324, Bonagrazia de Bergame fait précéder son examen des articles qui lui sont soumis par des considérations générales sur les «différentes raisons pour lesquelles un énoncé ou un dogme est jugé hérétique ». Cette déclaration liminaire propose un renversement complet du mode habituel de détection des hérésies. Au lieu de ne viser que le seul cas de paroles expressément contraires à des articles de foi, l'avocat franciscain propose d'instaurer un contrôle de la catholicité des énoncés qui tiendrait compte aussi bien de l'intention pure du locuteur que de la clarté de son expression. Des paroles ineptes ou équivoques sur des points concernant la foi pourraient alors suffire à [182] constituer un cas d'hérésie ${ }^{6}$. Cette extension vertigineuse du champ de la responsabilité doctrinale permettrait d'instaurer une censure efficace du travail exégétique. Pour sa part, Jacques Fournier propose une solution bien moins radicale. Argumentant de façon détaillée, il cherche à démontrer qu'Olivi et Joachim ont inventé un nouveau dogme - la rénovation de l'Eglise dans son sixième âge - dont la justification textuelle n'est pas probante. L'écart face à la tradition et la technique exégétique erronée ne suffisent pas à constituer l'hérésie. Celle-ci doit d'abord être repérée dans des noyaux dogmatiques clairement identifiables.

$\mathrm{La}$ règle générale invoquée pour condamner les excès de l'interprétation spirituelle mérite également que l'on s'y attarde un instant. L'expression est facilement reconnaissable puisqu'il s'agit d'une citation implicite du pseudo-Denys, transmise par l'intermédiaire de Thomas d'Aquin, qui subit toutefois une inflexion notable. Dans le prologue de son Commentaire des Sentences, Thomas disait en en effet plus précisément :

Ad destructionem autem errorum non proceditur nisi per sensum litteralem, eo quod alii sensus sunt per similitudines accepti et ex similitudinariis locutionibus non potest sumi argumentatio. Unde

\footnotetext{
${ }^{6}$ Bonagrazia da Bergamo, Allegationes super articulis tractis per dominum Papam de Postilla quam composuit frater Petrus Iohannis super Apocalipsim, Paris, BnF, lat. 4190, f. $40 \mathrm{v}$ : quod dictum sive dogma censetur hereticum pluribus modis [...] Quinto modo dicitur hereticum omne illud dictum quod sonat inordinatam locutionem circa ea que sunt fidei [...] Ut enim dictum censeatur catholicum duo requiruntur, scilicet quod catholica sit intentio proferentis et quod verba quod proferuntur sint congrua, clara, aperta et ordinata, ad fidei profexionem, et non incongrua, duplicia, oscura, vel inepta. »
} 
etiam Dionysius dicit in Epistola ad Titum quod 'symbolica theologia non est argumentativa, ${ }^{7}$

Durant les trois années qu'ils passèrent ensemble au studium de Cologne (1248-1251), Albert le Grand et Thomas d'Aquin ont consacré une part égale de leur attention au pseudo-Denys et à Aristote, l'un fournissant la matière des leçons de théologie et l'autre, celles de philosophie ${ }^{8}$. Ce que l'on décrit habituellement [183] comme le "tournant philosophique » des deux enseignants dominicains a simultanément été une plongée dans le corpus dionysien. Cette démarche ne devrait pas surprendre. Le platonicien chrétien anonyme $\mathrm{du} \mathrm{V}^{\mathrm{e}}$ siècle leur fournissait en effet des points d'appui pour penser l'articulation de la théologie et de la philosophie. L'une des pièces les plus intéressantes à cet égard est sa neuvième Epître dans laquelle Denys fait référence et synthétise l'enseignement de sa Theologia symbolica qui n'est jamais parvenue entre les mains des latins ${ }^{9}$. Lecteurs attentifs de l'ensemble du corpus dionysien, les deux dominicains ont été parmi les premiers, après Robert Grossesteste, à relever cette référence à un ouvrage perdu dont ils tentaient de deviner le contenu. Cependant, la règle mise en avant par Thomas ne constituait pas une originalité totale. Dans son cours, Albert le Grand la glosait en renvoyant à une règle similaire énoncée par Pierre Lombard: «Sed ex tropicis locutionibus non est recta argumentationis processio $»^{10}$. Retournant à Paris pour y accomplir ses actes d'accession à la maîtrise en théologie, Thomas d'Aquin eut une première occasion d'exposer le caractère scientifique de la théologie dans le prologue de sa lecture des Sentences. On entend clairement des échos dionysiens dans sa façon de présenter les différents modes d'expression théologique. Puisque les principes divins sont inaccessibles à l'esprit humain, à moins d'y être proportionnés au moyen de ressemblances sensibles, le recours au registre métaphorique est indispensable ${ }^{11}$. En revanche, l'établissement des

\footnotetext{
7 Thomas de Aquino, In I Sent., Prol., q. 1, art. 5, ed. Adriano Oliva, Les débuts de l'enseignement de Thomas d'Aquin et sa conception de la sacra doctrina, Paris, 2006, p. 331-332.

${ }^{8}$ A. Oliva, Ibid., p. 214-220, montre que c'est à Cologne que Thomas d'Aquin a pris en note sur le cours d'Albert sur la Hiérarchie céleste et sur les Noms divins, alors que l'on supposait habituellement que le premier cours aurait été donné à Paris avant le départ pour Cologne.

${ }^{9}$ Une hypothèse sur la nature de ce texte est formulée par István Perczel, « The PseudoDidymian De trinitate and Pseudo-Dionysius the Areopagite: A Preliminary Study », in Papers presented at the Sixteenth International Conference on Patristic Studies held in Oxford 2011. 6, Neoplatonism and Patristics (Studia Patristica, 58), Leuven, Peeters, 2013, p. 83-108.

${ }^{10}$ Albertus Magnus, Super Dionysii Mysticam theologiam et epistulas, ed. P. Simon, 1978 , p. 536, renvoyant à Petrus Lombardus, Sententiae, III, dist. 11, c. 2, ed. Brady, t. 2, p. 80.

${ }^{11}$ A. Oliva aurait pu signaler, p. 330, 1. 33, une citation implicite de Denys, De caelesti hierarchia, c. 1: «Quoniam neque possibile est ei qui secundum nos intellectui ad
} 
vérités doctrinales [184] ne peut se fonder que sur le seul sens historique. La destruction des erreurs et la réfutation des hérésies ne peut être conduite qu'au moyen d'une interprétation littérale de l'Ecriture.

Comme l'a souligné Beryl Smalley, cette expression proverbiale du primat du sens littéral a été largement repris dans l'école dominicaine et plus largement dans toute l'exégèse universitaire ${ }^{12}$. Il s'exprime généralement sous une forme dérivée, proche de celle qu'emploie Fournier : «Mystica theologia non est argumentativa ${ }^{13}$. La modification de la formulation d'origine, due à l'oubli du texte perdu, est un véritable contresens si l'on en juge à l'aune de la distinction des registres théologiques proposée par Denys. Elle se révèle pourtant très efficace pour qualifier une exégèse comme celle d'Olivi, pour qui le registre «mystique » correspond à l'interprétation allégorique.

L'œuvre exégétique de Pierre de Jean Olivi a fait l'objet d'une redécouverte très récente. Ce n'est à partir du volume publié en 1997 par Gedeon Gál et David Flood, Peter John Olivi on the Bible, que l'on a pu comprendre toute la richesse de sa lecture du texte sacré, grâce à l'édition critique de ses Principia in sacram scripturam et de son commentaire, bref mais incisif, sur Isaïe. Aux même dates, un livre magistral de David Burr a permis de prendre la mesure du joachimisme olivien, en invalidant définitivement l'atténuation de ce caractère qu'avait suggeré $\mathrm{R}$. Manselli ${ }^{14}$. Olivi est l'unique grand intellectuel universitaire médiéval qui ait accepté les principes de l'exégèse de Joachim et qui ait cherché à les développer. Il est en même temps un exégète universitaire de haut niveau, qui fait un usage abondant des outils de travail mis au point par ses prédécesseurs, qu'il s'agisse des correctoires bibliques ou de la Catena aurea de Thomas $\mathrm{d}^{\prime}$ Aquin ${ }^{15}$. La question qui se pose à la lecture des récentes [185] éditions de certains de ses grands commentaires bibliques ${ }^{16}$, ou de sa monumentale

immaterialem illam extendi caelestium hierarchiarum et imitationem et contemplationem, nisi ea quae secundum ipsum materiali manuductione utatur...», Chevallier ed., Dionysiaca, p. 735.

12 Jean de Paris (Quidort), De potestate regia et papali, ch. 15 (vérifier édition Bleienstein).

${ }^{13}$ Beryl Smalley, «Use of the 'Spiritual' Senses of Scripture in Persuasion and Arguments by Scholars in the Middle Ages », Recherches de Théologie Ancienne et Médiévale, 52, 1985 , p. 44-63.

${ }^{14}$ D. Burr, «The Persecution of Peter Olivi », Transactions of the American Philosophical Society, n.s., 66, part 5, 1976.

${ }^{15}$ L. J. Bataillon, Olivi utilisateur de la Catena Aurea de Thomas d'Aquin, in A. Boureau, S. Piron, dir., Pierre de Jean Olivi (1248-1298). Pensée scolastique, dissidence franciscaine et société, Paris, Vrin, p. 115-120.

${ }^{16}$ D. Flood, Peter of John Olivi on the Acts of the Apostles, St Bonaventure, 2001 ; D. Flood, Peter of John Olivi on Genesis, St Bonaventure, 2006 ; A. Boureau ed., Lecturae super Pauli Epistolas, Brepols, 2010 ; F. Iozzelli (ed.). Lectura Super Lucam et Lectura Super Marcum, Grottaferrata, 2010, pour ne citer que les textes les plus importants publiés ces dernières années. 
Lectura super Mattheum, éditée très partiellement ${ }^{17}$, est de comprendre comment il parvient à associer un niveau de lecture hyper-littérale à des envolées allégoriques justifiant sa théologie de l'histoire. Je voudrais ici tenter de dégager le principe herméneutique qui lui permet de réaliser une telle opération.

Le primat du sens historique est porté très loin chez Olivi. Pour ce qui est des écrits néo-testamentaires, il témoigne d'une sensibilité franciscaine attentive à l'historicité concrète de la vie du Christ, dont chacun des gestes peut être compris comme modèle d'une perfection évangélique. Dans l'interprétation qu'en donne Olivi, le vœu des frères mineurs les engage à reproduire ce modèle, dans la limite de leurs forces ${ }^{18}$. L'attention aux moindres détails de la vie du Christ commence dès la Nativité. On sait que le récit de l'évangile de Luc ne mentionne pas la présence de l'âne et du bœuf auprès de l'enfant Jésus. L'apparition des deux animaux est dû à un proverbe cité par Isaïe («Le bœuf reconnaît son propriétaire, et l'âne, la mangeoire de son maître », Is. 1,3), très tôt associé à cet épisode, aussi bien en raison de la référence à une mangeoire que de la suite de ce verset («Israël ne m'a pas reconnu »). Si, depuis Tertullien, les deux passages n'ont été associés qu'en un sens figuré dans l'exégèse savante, différentes représentations ont pourtant accepté de prendre à lettre cette présence animale $^{19}$. C'est en [186] particulier le cas de la célébration de la crèche par François d'Assise à Greccio, dans laquelle les animaux tenaient une place centrale. En exégète rigoureux, écartant systématiquement les récits apocryphes pour s'en tenir à la lettre du canon, Olivi ne peut y voir qu'une croyance infondée. Il justifie ce rejet par un argument littéral typiquement franciscain, attentif aux conditions matérielles concrètes de la scène : « Il n'est pas vraisemblable que Joseph ait amené le bœuf et l'âne, car il aurait été très cruel et inhumain, de sa part comme de la Vierge, de ne pas avoir vendu ses animaux ou d'autres, afin que le prix obtenu lui permette de louer une petite maison plus apte à accueillir le Seigneur, et d'acheter ou de louer des draps et d'autres choses nécessaires $»^{20}$. La pauvreté du Christ ne pouvait pas même s'accommoder du réconfort de ces bêtes pacifiques.

\footnotetext{
${ }^{17}$ Petrus Johannis Olivi, Lectura super Mattheum, prologus, S. Piron ed., Oliviana, 4, 2012 [online] http://oliviana.revues.org/498, mis en ligne le 14 mars 2013.

${ }^{18}$ D. Burr, Olivi and Franciscan Poverty. The Origins of the Usus Pauper Controversy, Philadelphia, 1989.

${ }^{19}$ L'une des plus anciennes traces d'acceptation littérale de la présence des animaux apparaît dans un texte copte du $\mathrm{VII}^{\mathrm{e}}$ siècle : K. Modras (ed.), Omelia copta attribuita a Demetrio di Antiocha sul natale e Maria vergine, Roma, CIM, 1994, p. 64-67.

${ }^{20}$ Lectura super Lucam, p. 248 «Non est autem uerisimile quod Ioseph sibi adduxisset bouem et asinum, tum quia tam ipse quam Virgo nimis impii et inhumani essent, si illa uel quecunque alia non uendidissent, ut ex pretio inde habito Christo possent domunculam aliquam magis aptam conducere ».
} 
La même exigence de stricte littéralité peut conduire Olivi à s'écarter d'une interprétation partagée par ses contemporains. C'est par exemple le cas pour le fameux verset Date mutuum, nihil inde sperantes (Luc 6,35) que les théologiens mobilisaient comme référence centrale de la condamnation de l'usure. Cet usage dérivait d'une décrétale d'Urbain III (ca. 1179), dans laquelle le pape avait cité ce verset pour expliquer de quelle façon un contrat de vente à terme méritait d'être qualifié d'usuraire, du seul fait de l'espoir d'un gain ${ }^{21}$. Le poids de cette autorité a conduit pendant plusieurs siècles les théologiens latins à employer ce verset pour appuyer la condamnation de l'usure pour laquelle le nouveau testament ne fournissait pas d'autre point d'appui $^{22}$. De façon attendue, l'exégèse médiévale de ce passage a suivi la même pente ${ }^{23}$. Pourtant, Olivi rejette cette [187] interprétation courante au motif qu'elle «exagère considérablement» la portée de ces mots du Christ $^{24}$. La méthode universitaire de la division du texte lui permet tout d'abord de replacer ce verset au sein du sermon dans la plaine. Il faut l'entendre dans une série d'injonctions surérogatoires qui, toutes, demandent aux chrétiens d'aller au-delà des préceptes de la justice que mêmes les gentils accomplissent. Correctement lu, dans son contexte, l'impératif est donc un conseil de perfection qui invite à prêter sans espoir d'être remboursé ${ }^{25}$. L'usure n'est condamnée qu'a fortiori, puisque la justice obligerait à rendre l'équivalent de la somme prêtée. Cette interprétation correspond effectivement au sens littéral du texte. C'est celle que défendait Ambroise ou Bède ${ }^{26}$. C'est également à cette solution que se range Calvin, estimant que « la sentence de Christ vulgairement estimée tres manisfeste $[\ldots]$ a esté faussement détournée $»^{27}$.

Un troisième exemple permettra de montrer que la même exigence s'applique également à la lecture de l'Ancien Testament. Au vingt-etunième chapitre de la Genèse, à la demande de Sara, Abraham chasse Hagar

${ }^{21}$ Consuluit (a. 1183-1185), X, 5, 19, 10, ed. Friedberg, c. 814.

22 Geraldus Odonis, De contractibus, in G. Ceccarelli, S. Piron, «Gerald Odonis Economics Treatise », Vivarium, 47, 2009, p. 196 : "Contra est verbum Salvatoris, Mutuum date nihil inde sperantes, ergo ex mutuo non potest aliquid ultra sperari, ergo multo minus recipi. »

${ }^{23}$ Albertus Magnus, Ennarationes in primam partem evang. Lucae, ed. Borgnet, Paris, 1894, p. 436. Bonaventura, Commentarius in Evangelium S. Lucae (Opera Omnia, t. 7), Quaracchi, 1895, p. 157-158

${ }^{24}$ Pierre de Jean Olivi, Traité des contrats, ed. S. Piron, Paris, 2012, p. 182 : «quidam nimium exaggerantes verbum Christi ... »

${ }^{25}$ Petrus Iohannis Olivi, Lectura super Lucam, ed. F. Iozzelli, Grottaferrata, 2010, p. 344 :

« Nota etiam quod hic proprie loquitur aut de supererogativa perfectione dandi mutuum, aut de illa que sic fit principaliter propter Deum, quod non figit finem suum, nec suam finalem spem $\mathrm{n}$ redditione equivalentis, sed potius in premio vite eterne $»$.

${ }^{26}$ Ambrosius, Expositio evangelii secundum Lucam, ed.C. Schenkl, Wien, CSEL, p. 211 ; Beda, In Lucae evangelium expositio, ed. D. Hurst, Turnhout, 1960, p. 145-146.

${ }^{27}$ Jean Calvin, Lettre à Claude de Sachins, in Opera omnia, Brunswick, 1871, t. 10, c. 245 246. 
et l'enfant qu'elle a eu de lui, afin qu'Isaac demeure son seul héritier. Dans l'Epitre aux Romains, Paul fournit de cet épisode une interprétation allégorique, distinguant les "enfants de la promesse » des enfants de la chair (Rm 9,1-29). Dans son commentaire de la Genèse, Olivi s'interrroge sur ce point. Cela signifie-t-il à la lettre que personne de la descendance d'Ismaël ne sera sauvé et que seule la postérité d'Isaac le sera ? Après avoir noté que seule une interprétation de type allégorique peut justifier une telle séparation entre fils de la chair et fils de la grâce, Olivi donne pourtant un argument [188] historique ${ }^{28}$. C'est parce que le culte divin allait être confié au peuple issu d'Isaac qu'il aurait été nocif et dangereux qu'il se mélange à d'autres nations.

Pour comprendre la façon dont Olivi parvient à s'approprier l'exégèse joachimite tout en demeurant un exégète scrupuleusement respectueux du sens littéral, il faut à nouveau se tourner vers le Pseudo-Denys. Comme Thomas, Olivi considère Denys comme un maître d'exégèse. Les écrits les plus importants à considérer figurent dans ses principia bibliques, ainsi que dans le deuxième chapitre de son commentaire de la Hiérarchie céleste ${ }^{29}$. Le point crucial de l'herméneutique qu'il en déduit concerne la nécessité des figures dissemblables qui ont pour double fonction de montrer et de cacher simultanément, à la façon d'un nuage qui ne permet d'affronter la lumière du soleil qu'en la recouvrant d'un voile. Cette monstration est l'unique moyen par lequel la divinité peut s'exprimer, par la médiation de paroles appropriées à une compréhension par l'intellect humain qui suggèrent aussi, inséparablement, qu'elles transmettent un sens spirituel plus élevé. La transcendance du message divin n'est accessible que dans l'élément du langage ; la tâche de l'exégète est de le déchiffrer comme en un miroir dans lequel se reflète la lumière spirituelle.

Pour décrire le règle herméneutique que met en œuvre Olivi, on peut ainsi parler d'une « réversibilité de la lettre $»^{30}$. Le sens spirituel n'est pas à chercher au-delà de la lettre, dans un niveau supérieur qui réclamerait l'abandon de toute dimension charnelle pour atteindre l'esprit pur, comme dans le modèle classique de l'allégorie. Au contraire, ce sens est déjà contenu et caché dans l'expression littérale. Dans un développement [189] spectaculaire, l'un des principia présente le Christ comme medium de

\footnotetext{
${ }^{28}$ Olivi on Genesis, p. 387 : «Dicendum quod non potest hic ad plenum sufficiens ratio dari nisi recurratur ad mysticam rationem ... Pro tanto tamen hic est ratio litteralis , quia populus in quo erat illo tempore cultus Dei principaliter servandus et propagandus, erat de solo Isaac nasciturus: illi autem populo erat valde nociva et periculosa commixtio ceterorum si starent cum eis ut concives et cohaeredes; quia tunc de facili scinderent et corrumperent veritatem divini cultus. »

${ }^{29}$ Olivi on the Bible, p. 104-108.

${ }^{30} \mathrm{~S}$. Piron, « Le métier de théologien », p. 56-83.
} 
l'Ecriture sainte - tout à la fois milieu, moyen et médiateur qui permet de dévoiler le sens d'un texte clos et scellés ${ }^{31}$. C'est le principe même de l'incarnation qui est mis en jeu dans cette opération : le sens spirituel s'est véritablement incarné dans la lettre, de la même façon que la divinité s'est incarnée en un homme. Pour l'exégète franciscain, l'Ecriture sainte est bien une chair habitée par l'esprit. De même que l'on ne va au Père que par le Fils, le sens spirituel ne peut être atteint qu'à partir du sens littéral et grâce à lui. La compréhension la plus aboutie est donc celle qui parvient à saisir leur union intime.

Le prologue de la Lectura super Mattheum apporte un témoignage majeur de cette herméneutique fondée sur une théologie de l'incarnation ${ }^{32}$. Une série organisée de six questions propose en guise de préambule un remarquable exercice de critique biblique. Au lieu de chercher à dépasser et annuler la discordance entre les quatre narrations évangéliques, Olivi cherche plutôt à comprendre la raison de cette diversité. Plusieurs types d'arguments lui permettent de produire une véritable déduction de la nécessité d'une transmission plurielle du message divin. Le Christ ne pouvait pas écrire lui-même ou dicter sa doctrine, sans contrevenir à sa propre humilité. S'il devait être rédigé par d'autres, le récit de sa vie ne pouvait être transmis avant l'accomplissement de la Passion et de la Résurrection. Une pluralité de témoins était indispensable pour assurer la crédibilité du message, quatre étant le nombre le plus stable. Pour le même motif, les différents narrateurs ne pouvaient pas répéter exactement les mêmes faits, une marge de variation offrant la garantie que leur convergence de fond n'était pas le produit d'un collusion suspecte. Mais la raison essentielle de leurs discordances est qu'aucun d'entre eux ne pouvait épuiser, à lui seul, la totalité des faits et gestes du Christ :

Igitur si omnes penitus idem dixissent, credi posset quod omnia Christi magnalia narrassent, quod quidem non esset [190] honorificum Christo, nec utile sublevationi nostre speculationis in infinitam abyssum magnalium $\mathrm{Christi}^{33}$.

La pluralité et la divergence des récits permettent ainsi de manifester l'insuffisance de témoignages rédigés dans un langage humain et l'excès de sens des actes du Sauveur. La lettre écrite vaut d'abord comme trace conduisant à une présence. Mais il faut également rappeler que celle-ci, de surcroît, a refusé de se livrer en toute transparence: souvent muette,

\footnotetext{
${ }^{31}$ Peter of John Olivi on the Bible, p. 78-123.

${ }^{32}$ S. Piron, « L’herméneutique évangélique olivienne », Oliviana, 4, 2012, http://oliviana.revues.org/765

${ }^{33}$ Petrus Johannis Olivi, Lectura super Mattheum, prologus, ed. S. Piron, Oliviana, 4, 2012, http://oliviana.revues.org/498, § 95 .
} 
s'exprimant sur un mode énigmatique, préférant parfois se faire comprendre par des gestes, elle a manifesté de la sorte son appartenance à l'humanité, définie par la corporéité et non pas l'intelligibilité immédiate des substances spirituelles. Ce n'est donc pas uniquement la faiblesse d'un texte pluriel, présentant des contradictions apparentes, qui rend indispensable un travail exégétique d'excavation du sens spirituel. L'opacité de la chair dans laquelle la divinité s'est incarnée est à la fois la raison de l'obscurité principielle du texte et le moyen de son éclaircissement.

Dicté par l'Esprit saint, le texte sacré est intégralement signifiant. Cependant, sa richesse de sens n'est pas répartie de façon égale en chaque lieu du texte. L'exégète doit au contraire chercher les lieux spécifiques où le texte peut s'ouvrir, à l'image de la pierre du sépulcre de Lazare, pris en exemple et modèle dans les Principia $^{34}$. Il sait repérer les grains d'or dispersés dans le sable que d'autres avant lui n'avaient pas su voir ${ }^{35}$. Une telle démarche réclame un rôle actif de la part de l'interprète, chargé de découvrir un réseau de sens qui demeure caché au regard d'un œil non averti. On peut à bon droit parler à ce propos de «saut herméneutique » ${ }^{36}$. Il est d'ailleurs remarquable qu'Olivi insiste sur l'instantanéité de ce dévoilement. Parlant des déductions que permet la compréhension du Christ comme medium de [191] l'Ecriture, il souligne que l'intelligence spirituelle $\mathrm{du}$ texte est donnée aussitôt (statim $)^{37}$. Aucune inspiration particulière de l'exégète ne semble requise. Cependant, le même vocabulaire est employé pour décrire l'inspiration subite par laquelle Joachim a pu, d'un coup, comprendre la concorde de l'Ancien et du Nouveau Testament ${ }^{38}$. Il faudrait en conclure qu'Olivi attend que l'exégète, se saisissant de la clé fournie par Joachim, vive une expérience comparable de compréhension instantanée. Or, et c'est le point crucial de toute cette élaboration, la clé en question repose sur une correspondance littérale entre l'histoire biblique et celle de l'Eglise. En d'autres termes, c'est bien une clé d'interprétation littérale qui ouvre, d'un seul coup, la compréhension spirituelle du texte sacré.

L'usage que fait Olivi du terme litteralior me semble particulièrement significatif de son effort d'appréhension synthétique du spirituel et du

\footnotetext{
${ }^{34}$ Olivi on the Bible, p. 59.

${ }^{35}$ Lectura super Isaiam, in Olivi on the Bible, p. 198-199 : « aliquando quis advertit aliqua grana auri in arena, quae non alius, quamvis eadem loca in generali aspiciat ... et iste est unus modus per quem Deus subito docet maxima de Scripturis sacris ».

${ }^{36}$ Gilbert Dahan, «L'exégèse des livres prophétiques chez Pierre de Jean Olieu », in A. Boureau, S. Piron (dir.), Pierre de Jean Olivi (1248-1298). Pensée scolastique, dissidence spirituelle et société, Paris, Vrin, 1999, p. 91-114.

${ }^{37}$ Olivi on the Bible, p. 89 : « Statim in medio summae abstractionis aperitur omnis materialitas...».

${ }^{38}$ Olivi on the Bible, p. 197-198.
} 
littéral. Une interrogation des bases de données textuelles disponibles permet de vérifier que l'usage du comparatif est relativement rare ${ }^{39}$. Albert le Grand est l'un des premiers auteurs qui l'emploie, dans certaines situations qui l'amènent à privilégier, entre deux solutions recevables, celle qui offre «le sens le plus littéral ${ }^{40}$. Quelques sondages montrent que le terme a pu servir dans les décennies suivantes à exprimer la règle du primat du sens littéral ${ }^{41}$. Cependant, Olivi paraît bien [192] être le commentateur le plus attaché à l'usage de ce terme, aussi bien en nombre d'emplois qu'en raison de la valeur particulière qu'il lui confère.

Dans un chapitre de la Lectura super Mattheum qu'a édité par le franciscain australien Tom Murtaugh, l'interprétation du syntagme pauperes spiritu (Mat. 5,3) donne lieu à une divergence entre deux interprétations. Selon Augustin et Chrysostome, sous la désignation des «pauvres d'esprit», il faudrait entendre les humbles qui n'ont pas l'esprit gonflé d'orgueil. En revanche, pour Basile de Césarée, le terme désigne ceux qui se sont rendus pauvres en suivant les conseils évangéliques de vendre tous ses biens. Cette lecture paraît plus littérale, et donc plus rigoureuse, puisqu'elle permet de donner un sens fort au terme spiritus, compris comme volonté spirituelle de pauvreté ${ }^{42}$. Ce sens est également préférable en raison de sa valeur englobante, puisque cette pauvreté inclut et exige l'humilité, et dispose à l'atteindre.

Dans le commentaire de l'Ecclésiaste, le fait que «le vent (spiritus) reprenne ses tours » pose une difficulté, puisque les mouvements de l'air paraissent être davantage linéaires que circulaires. Certains voudraient

\footnotetext{
${ }^{39}$ Dans la base de données « The Library of Latin Texts - Series A et B » (interrogée le 15 octobre 2013), Olivi est le seul auteur qui emploie les formes litteralior ou litteralius. Je note un unique emploi chez Denys le Chartreux, Commentaria in psalmos, art. 93.

40 Albertus Magnus, Ennarationes in evangelium Lucae, ed. A. Borgnet, Paris, Vivès (Opera Omnia, 22), 1894, p. 33 : «Et haec expositio notanda est quia illa sola omnibus aliis est verior, et litteralior» ; Id., Ennarationes in evangelium Matthaei (Opera omnia, 21), Paris, 1893, p.193 : «Sed prima expositio litteralior est, et melior ». Une interrogation de la base «Alberti Magni E-Corpus » de l'université de Waterloo donne encore deux résultats dans le Super Marcum (ed. Borgnet, p 562 et 586) et Super Iohannem (ed. Borgnet, p. 68).

${ }^{41}$ Petrus Aureoli, Notationes in universam sacram scripturam, Parisiis, apud R. Foüet, 1610, p. 69 : « Et ideo melius est et litteralius dividere eo modo quo dictum est ».

${ }^{42}$ Petrus Johannis Olivi, Lectura super Mattheum, 5, 3, Paris, BnF lat. 15588, f. 41rb, ed. T. F. Murtaugh, Peter Olivi's Matthew commentary : a critical edition of chapter 5, verses 126 with a commentary, Melbourne College of Divinity, 1992: " per 'pauperes spiritu', secundum Augustinum et Chrysostomum, 'intelliguntur humiles, qui parum habent de inflante spiritu'. Secundum vero Basilium in Regula sua, capitulo 205, intelliguntur hi 'qui non alia causa pauperes sunt nisi propter doctrinam Domini' dicentis: Vade et vende omnia quae habes et da pauperibus. Et secundum hoc 'spiritu' sumitur pro voluntate spirituali et fervida, ut sit sensus 'pauperes spiritu', id est 'pauperes ex voluntate seu voluntarii', et hic modus est litteralior, quamquam primus in hoc includatur, tum quia paupertas maximam abiectionem mundani honoris et tumoris in se ipsa includit, tum quia ad ipsam vehementer disponit, tum quia ipsam supra modum exigit. Unde omni homini abominabile est videre pauperem superbum, et praecipue pauperes Christi. »
} 
échapper à la difficulté en appliquant la phrase au soleil, sujet de la phrase précédente. Mais le mode le plus littéral est celui qui parvient à rendre compte, à la lettre, de l'occupation de l'espace par un vent qui rencontre des obstacles et se trouve réfléchi dans l'ensemble de la région où il souffle ${ }^{43}$. [193]

L'ouvrage dans lequel Olivi a le plus abondamment recours à ce terme est la Lectura super Apocalipsim. L'un des passage les plus intéressants est Ap. 7, 4, qui donne l'occasion de formuler une règle de méthode. Il peut arriver que l'accomplissement des prophéties au sens allégorique précède leur accomplissement littéral. C'est par exemple le cas lorsque Malachie annonce qu'Elie sera envoyé « avant que vienne le jour du Seigneur » (Mal 4, 5-6). Certes, le Christ a lui-même annoncé que la prophétie s'était accomplie en Jean Baptiste (Mt 17, 11-1). Mais cet accomplissement n'était que spirituel, puisqu'un accomplissement littéral est attendu dans un retour d'Elie ${ }^{44}$. «Or, quand le sens le plus littéral se rapporte aux biens ou aux actes derniers, il est de ce fait plus spirituel que ne le sont les sens allégoriques dont l'accomplissement le précède ». Faisant appel au PseudoDenys, Olivi souligne alors que Dieu est plus proprement nommé par des noms abstraits que par des figures sensibles; celles-ci ne sont qu'allégoriques, alors que les premiers sont à la fois plus littéraux et plus spirituels.

La règle qui émerge de ce passage n'a qu'une valeur ponctuelle. Son efficacité mérite toutefois qu'on lui accorde un statut plus général de règle d'interprétation centrale dans l'exégèse olivienne. On peut en trouver confirmation en notant que la même conjonction des opposés s'exprime, dans des termes convergents, dans la question sur la très haute pauvreté : c'est lorsqu'elle se présente dans le plus grand dénuement que la divinité du Christ apparaît le mieux comme principe spirituel [194] dégagé de toute

\footnotetext{
${ }^{43}$ Petrus Iohannis Olivi, Lectura super Ecclesiasten, ed. J. Schlageter, Grottaferrata, 2003, p. 93 : «Sed primus modus uidetur esse litteralior ».

${ }^{44}$ Lectura super Apocalypsim, ed. W. Lewis, « Nec mireris si sensus allegoricus, quoad impletionem in effectu, precedat hic litteralem, quia hoc alibi invenies. Quod enim Malachie ultimo dicitur Helias mittendus antequam veniat dies Domini, dicit autem Christus Matthei XVII ${ }^{\circ}(\mathrm{Mt})$ hoc iam impletum esse in Iohanne Baptista, et nichilominus ad litteram implendum esse in ipso Helia. Constat autem quod Iohannes non fuit Helias nisi mistice et allegorice. Sepe etiam a Christo et a prophetis dicuntur plura litteralius respicientia statum eterne glorie vel extremi iudicii, que tamen allegorice prius implentur in precursoriis gratiis vel iudiciis. Attamen quando litteralior sensus sic respicit finaliora bona vel facta, tunc ipse est spiritualior quam sint allegorici ipsum precurrentes, iuxta quod litteralius [et] magis proprie dicitur Deus esse vita et sapientia et summum bonum quam dicatur esse leo vel sol vel ros et mel, et tamen primum litteralius dictum est spiritualius et perfectius quam sit secundum dictum, quod est translativum et allegoricum ».
} 
implication dans les choses de ce monde ${ }^{45}$. Cette pauvreté constitue bien le point de passage du littéral au spirituel, aussi bien théologiquement qu'en matière d'exégèse. Si cette règle est limpide, elle n'est en réalité accessible qu'au moyen d'une première décision, un acte de foi qui permet de faire basculer une théologie de l'incarnation en théologie de l'histoire. Toute la démarche suivie par Olivi suppose d'accepter l'identification de François d'Assise comme rénovateur de la pauvreté évangélique, ouvrant le sixième âge de l'Eglise. C'est là sa grande faiblesse. Pour le suivre, il faut en effet accepter un grand nombre de présupposés, aussi bien exégétiques qu'ecclésiologiques. Pour un lecteur extérieur à cet univers interprétatif, comme l'était Jacques Fournier, cette exégèse n'est que folie, dépourvue de toute garantie de la raison ou de l'autorité des Pères.

Cependant, pour l'historien de l'exégèse, la question ne s'arrête pas là. Les procédures employées ne relèvent pas d'un pur arbitraire. Comme Fournier le notait également, Joachim et Olivi font un usage exagéré de toutes les indications chiffrées qu'ils trouvent dans le texte sacré. C'est bien là qu'ils ont trouvé un point d'appui à leur spéculation. Si l'Ecriture sacrée transmet un message crypté, il est compréhensible que la capacité symbolique particulière chiffres ait stimulé l'imagination exégétique. Au cours de ces dernières décennies, une attention nouvelle a été accordée à l'exégèse visuelle, aussi bien pour Joachim que d'autres auteurs du XIIe siècle $^{46}$. Il ne serait pas injustifié que l'exégèse numérologique bénéficie à présent de mêmes faveurs.

\footnotetext{
${ }^{45}$ Quaestio de altissima paupertate, in Johannes Schlageter, Das Heil der Armen und das Verbreden der Reichen, Werl, 1989, p. 135 : «Si Christus non ostendisset se tamquam summum contemptorem et conculcatorem rerum, non plene ostendisset suum spiritualissimum dominium quo superfertur omnibus, etiam secundum humanitatem ». ${ }^{46}$ Marco Rainini, Disegni dei tempi. Il Liber Figurarum e la teologia figurativa di Gioacchino da Fiore, Roma, Viella, 2006.
} 